\title{
Total thyroidectomy in refractory amiodarone induced thyrotoxicosis: a case series of 12 patients
}

\author{
T. Drescher ${ }^{1}$, T. Clerici2 ${ }^{2}$ W. Kolb², M. Brändle ${ }^{1}$, S. Bilz ${ }^{1}$ \\ ${ }^{1}$ Division of Endocrinology, Diabetes and Osteology, Kantonsspital St. Gallen \\ 2 Department of Surgery, Kantonsspital St. Gallen
}

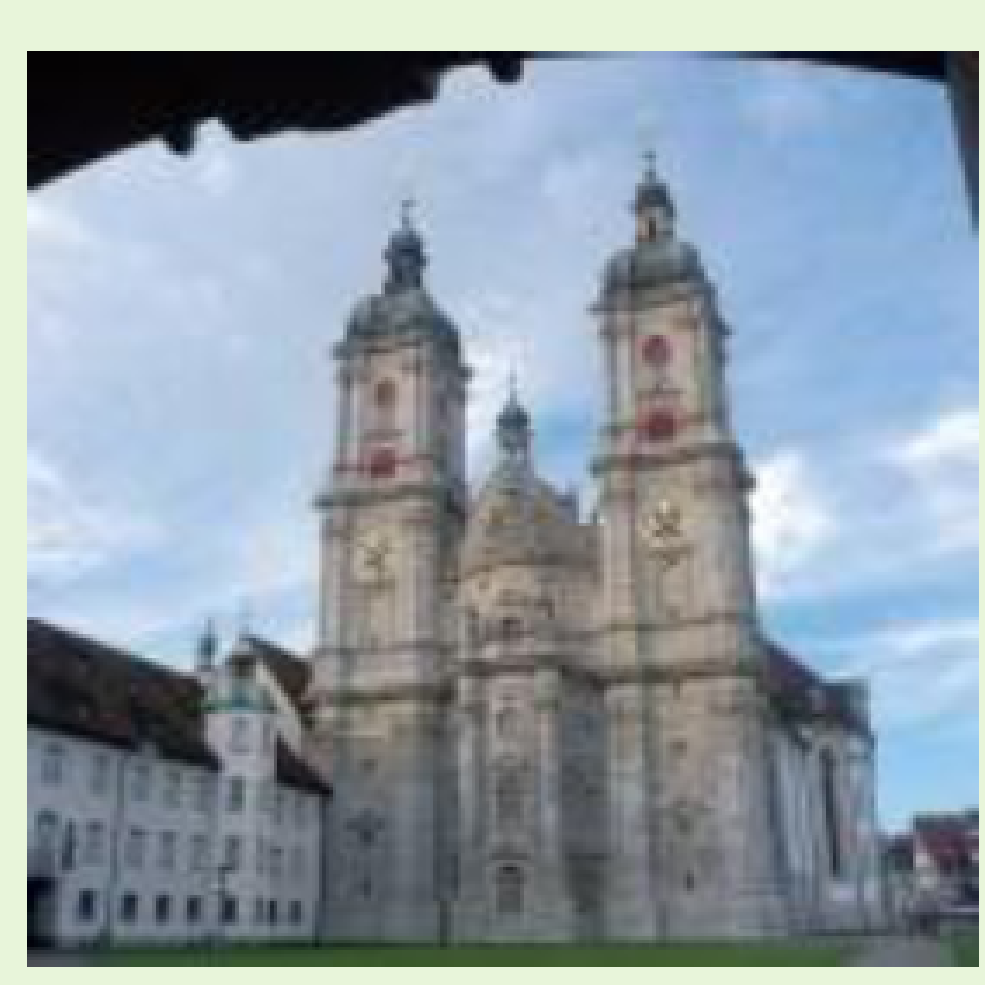

\section{Introduction:}

Amiodarone induced thyrotoxicosis (AIT) develops in $5-10 \%$ of patients and may occur at any time throughout the course of treatment including months after discontinuation. Two distinct forms of AIT are distinguished and treated differently. lodine-induced hyperthyroidism, typically seen in patients with underlying thyroid disease, is referred to as type 1 AIT and treated with high doses of thionamide antithyroid drugs and perchlorate. Type 2 AIT is a destructive thyroiditis and most cases respond to highdose glucocorticoids. However, mixed forms and refractory cases are occasionally observed and prolonged hyperthyroidism may lead to substantial morbidity, especially in patients with significant cardiac comorbidities. Thyroidectomy rapidly restores normal thyroid function but must be performed in overtly hyperthyroid often critically ill patients.

\section{Methods:}

Retrospective analysis of the clinical records of all 12 patients with AIT who underwent total thyroidectomy at the department of surgery, Kantonsspital St. Gallen, since 2006

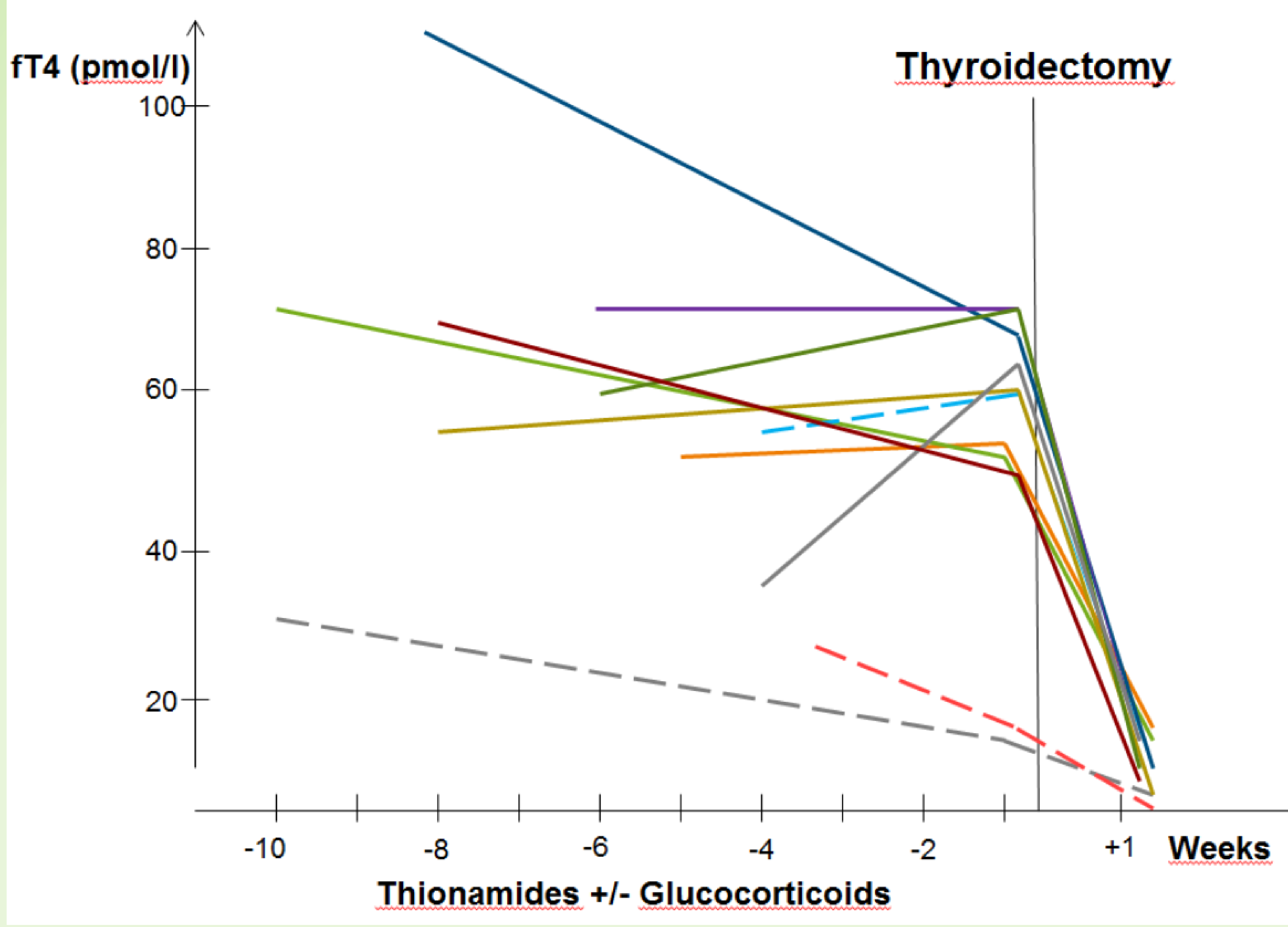

Figure: course of thyrotoxicosis according to fT4 values under medical treatment with high-dose thionamides alone (dotted lines) or in combination with glucocorticoids (continuous lines), 3-10 weeks before and 1 week after thyroidectomy

\begin{tabular}{|c|c|c|c|c|c|c|c|c|c|c|}
\hline $\begin{array}{l}\text { Patient } \\
\text { number }\end{array}$ & Sex & Age & Heart disease & LV-EF & ICD & $\begin{array}{l}\text { Duration of amiodarone } \\
\text { therapy, years }\end{array}$ & Thyroid ultrasound & $\begin{array}{c}\text { Thyroid } \\
\text { antibodies }\end{array}$ & $\begin{array}{c}\text { Antithyroid drug } \\
\text { therapy } \\
\end{array}$ & $\begin{array}{l}\text { Hospital stay / ICU, } \\
\text { days }\end{array}$ \\
\hline 1 & $\mathrm{~F}$ & 64 & Hypertensive & $50 \%$ & $\mathrm{~N}$ & 3 & Diffuse goiter & Neg. & PTU + Pred & $4 /-$ \\
\hline 2 & $M$ & 49 & Dilated CMP & $30 \%$ & $Y$ & 2 & Diffuse goiter & Neg. & PTU + Pred & $2 /-$ \\
\hline 3 & $M$ & 57 & Arrhythmogenic & $50 \%$ & Y & 2 & normal & Neg. & PTU + Pred & $2 /-$ \\
\hline 4 & $M$ & 50 & $\mathrm{CHD}$ & $45 \%$ & Y & $>4$ & Diffuse goiter & Neg. & PTU + Pred & $5 / 2$ \\
\hline 5 & $M$ & 67 & $\mathrm{CHD}$ & Not known & Y & Not known & Diffuse goiter & Not known & Not known & $5 /-$ \\
\hline 6 & $M$ & 61 & $\mathrm{CHD}$ & $35 \%$ & $\mathrm{Y}$ & 4 & Diffuse goiter & Neg. & CMZ + Pred & $2 /-$ \\
\hline 7 & $\mathrm{~F}$ & 81 & Hypertensive & $25 \%$ & $\mathrm{~N}$ & weeks & Nodular goiter & Not known & $\mathrm{CMZ}+$ Perchlorate & $20 / 20$ \\
\hline 8 & $M$ & 63 & Not known & $30 \%$ & Y & 4 & normal & Neg. & $\mathrm{CMZ}+$ Pred & $4 /-$ \\
\hline 9 & $M$ & 64 & Valvular & $35 \%$ & $\mathrm{~N}$ & 3 & normal & Neg. & $\mathrm{CMZ}+$ Pred & $3 /-$ \\
\hline 10 & $M$ & 51 & Dilated CMP & $25 \%$ & $Y$ & 1 & Diffuse goiter & Neg. & Pred & 4/- \\
\hline 11 & $\mathrm{~F}$ & 61 & Hypertensive & $50 \%$ & $\mathrm{~N}$ & 2.5 & Diffuse goiter & Neg. & $\mathrm{CMZ}+$ Pred & $4 /-$ \\
\hline 12 & $M$ & 52 & CHD & $20 \%$ & $Y$ & 3 & normal & Neg. & CMZ + Pred & $3 / 1$ \\
\hline
\end{tabular}

CMP, cardiomyopathy; CHD, coronary heart disease; LV-EF, left ventricular ejection fraction; ICD, implanted cardioverter defibrillator; PTU, Propylthiouracil; CMZ, Carbimazole; Pred, Prednisone

\section{Results/Cases:}

The age of the patients ranged from 50-81 years and 3 were female. All patients had an underlying cardiac disease and 8 had an ICD. Thionamides, glucocorticoids, or both had been administered for 3-10 weeks prior to surgery. Indications for thyroidectomy included unresponsiveness to medical treatment in all cases and worsening of the underlying cardiac condition in several cases. Euthyroidism was restored quickly in all subjects. The length of the postoperative hospital stay ranged from 2-5 days and temporary intensive care was required in 3 patients. One patient died 3 weeks after surgery because of multiple preexisting complications. All other patients were euthyroid on levothyroxine replacement and in stable or improved cardiac condition for at least 1 year.

\section{Discussion:}

The medical management of AIT remains to be challenging since even high-dose thionamides need to be administered over a long period to restore normal thyroid function in patients with iodine-induced hyperthyroidism (type $1 \mathrm{AIT}$ ), a subset of patients with amiodarone induced thyroiditis (type 2 AIT) poorly responds to high dose steroids and even mixed forms may occur. Therefore, euthyroidism may not rapidly be achieved in a considerable proportion of patients at high risk of adverse cardiac outcomes. Thus, surgical management is a viable option but seems to be rarely performed due to the anticipated high perioperative risk. However, this risk has to be balanced with the well described detrimental effects of persistent thyrotoxicosis. AIT is a predictor of adverse cardiovascular outcomes, particularly in patients with reduced left ventricular function. The few case series on thyroidectomy in AIT available from the literature, in agreement with our results, report an overall low complication rate. Moreover, in addition to the immediate restoration of euthyroidism, cardiac function improved significantly in a substantial portion of the reported subjects. The prerequisite for such a successful course is a close multidisciplinary collaboration between endocrinologists, cardiologists, anesthesists and endocrine surgeons. Referral for thyroidectomy should rather be considered when failure of medical treatment is anticipated than when it has occurred.

\section{Conclusion:}

Total thyroidectomy is an effective and save treatment in patients with AIT unresponsive to medical therapy and should be considered early to prevent subsequent cardiac morbidity due to prolonged overt hyperthyroidism.

\section{References:}

Cohen-Lehman J. et al. Effects of amiodarone therapy on thyroid function. Nat. Rev. Endocrinol. 2010; 6: 34-41 - Bogazzi F. et al. Approach to the patient with Amiodarone-induced thyrotoxicosis. J. Clin. Endocrinol. Metab. 2010; 95: 2529-35

- Tomisti L. et al. Total thyroidectomy in patients with amiodarone-induced thyrotoxicosis and severe left ventricular systolic dysfunction. J. Clin. Endocrinol. Metab. 2012; 97: 3515-21

- Gough J. and Gough I.R. Total thyroidectomy for amiodarone-associated thyrotoxicosis in patients with severe cardiac disease. World J. Surg. 2006; 30: 1957-61 